of these associations needs to be elucidated and further research is necessary to assess whether other measures of physical capability, including chair rises and balance, have similar predictive value.

\section{Ageing \\ 069 THE IMPACT OF CATARACT SURGERY ON HEALTH RELATED QUALITY OF LIFE AND TIME USE IN KENYA, BANGLADESH AND THE PHILIPPINES}

${ }^{1} \mathrm{~S}$ Polack, ${ }^{2} \mathrm{C}$ Eusebio, ${ }^{3} \mathrm{~W}$ Mathenge, ${ }^{4} \mathrm{Z}$ Wadud, ${ }^{5} \mathrm{AKM}$ Mamunur, ${ }^{1} \mathrm{~A}$ Foster, ${ }^{1} \mathrm{H}$ Kuper. 'London School of Hygiene \& Tropical Medicine, Keppel Street, London, UK; ${ }^{2}$ Cataract Foundation of the Philippines, Bacolod, Philippines; ${ }^{3}$ Ritt Valley Provincial Hospital, Nakuru, Kenya; ${ }^{4}$ Child Sight Foundation, Dhaka, Bangladesh; ${ }^{5}$ CSS Rawm Hospital, Khulna, Bangladesh

\section{doi:10.1136/jech.2009.096727q}

Objective: To explore the impact of cataract surgery on health related quality of life (HROoL) and time-use among adults aged $\geqslant 50$ years Kenya, The Philippines and Bangladesh.

Methods: This was a multi-centre intervention study. Across the three countries at baseline, 651 population-based cases aged $\geqslant 50$ years visually impaired from cataract (visual acuity in the better eye $<6 / 24$ ), and 561 age-gender-cluster-matched controls with normal vision were identified. All participants were interviewed in their homes about vision related quality of life (WHO/PBD VF20), generic HRQoL (Euroqol) and time-use. Cases were offered free/ subsidised cataract surgery. Approximately one year later participants were re-interviewed using the same questionnaires. Response rate at follow up was $84 \%$ for operated cases, and $80 \%$ for controls. Results: At baseline, cases had substantially poorer vision specific and generic HRQoL compared to controls. Cases were also spent significantly less time on productive activities (paid and non-paid work) and more time in inactivity. Approximately one year after cataract surgery, mean vision specific and generic HRQoL improved $(p<0.001)$ to the level of controls with normal vision. Effect sizes for change in VROoL were large $(>1.0)$ regardless of pre-operative VA, but were larger for those who had perception of light at baseline and for people who were operated in both eyes. Poor VA outcome from surgery was a constraint to achieving optimal post-operative VRQoL. At follow-up, operated cases were more likely to undertake and spent 12 hours more on productive activities compared to baseline $(p<0.001)$. Time spent in "inactivity" in Kenya and Bangladesh decreased by approximately 2 hours. Frequency of reported assistance with activities was more than halved in each setting among operated cases $(p<0.001)$. Conclusion: Using three different outcome measures, this study demonstrated positive impacts of cataract surgery on the lives of older adults in three low-income settings, which has advocacy implications for blindness prevention programs. The observed increased time spent on productive activities, reduced time in inactivity and reduced assistance has positive implications for wellbeing and inclusion and supports arguments of economic benefit at the household level from cataract surgery.

\section{POVERTY AND BLINDNESS: AN INTERVENTION STUDY TO ASSESS THE IMPACT OF CATARACT SURGERY ON POVERTY}

${ }^{1} \mathrm{H}$ Kuper, ${ }^{2} \mathrm{~S}$ Polack, ${ }^{3} \mathrm{C}$ Eusebio, ${ }^{2} \mathrm{~W}$ Mathenge, ${ }^{4} \mathrm{Z}$ Wadud, ${ }^{4} \mathrm{R}$ Mamun, ${ }^{2} \mathrm{~A}$ Foster. ${ }^{1}$ Department of Epidemiology and Public Health, London School of Hygiene \& Tropical Medicine, London, UK; ${ }^{2}$ Department of Infectious and Tropical Disease, London School of Hygiene \& Tropical Medicine, London, UK; ${ }^{3}$ Cataract Foundation of the Philippines, Bacolod, the Philippines; ${ }^{4}$ Child Sight Foundation, Dhaka, Bangladesh

doi:10.1136/jech.2009.096727r

Objective: To assess the association between poverty and visually impairing cataract, and the impact of cataract surgery on alleviating poverty.
Design: Multicentre intervention study.

Setting: Centres in Kenya, Bangladesh and the Philippines. Baseline assessment was in 2005-2006, with follow-up one year later.

Participants: We recruited 596 cases and 481 controls, aged $\geqslant 50$ years. Cases had visual acuity (VA) $\leqslant 3 / 36$ in the better eye due to cataract. Controls were age-sex-community-matched to cases with normal vision (VA $\geqslant 6 / 18)$. Controls and most cases (65\%) were recruited through population-based surveys, with additional cases $(35 \%)$ identified through population case finding. 413 cases underwent cataract surgery. At follow-up, we re-interviewed 345 operated cases and 552 controls.

Interventions: Cataract surgery was offered to cases.

Main outcome measures: Poverty was measured through: (1) per capita household expenditure (PCE), (2) household assets and (3) household self-rated wealth.

Results: At baseline, operated cases were significantly more likely than controls to be in the lowest quartile of PCE in Kenya (OR 3.5, 95\% CI 1.1 to 11.2 ), Bangladesh (3.0, 1.5 to 6.1) and the Philippines (4.4, 1.9 to 10.3), with a significant dose-response relationship across quartiles of PCE. The pattern was similar for assets and selfrated wealth. At follow-up, PCE had increased significantly among the operated cases in all countries, but not among the controls. Operated cases were no longer more likely than controls to be in the lowest category of PCE in Kenya and the Philippines, and the association in Bangladesh was weakened (2.0, 1.0 to 4.0). Operated cases remained poorer than controls in terms of assets and self rated wealth, although the association was weaker than at baseline.

Conclusion: The Cataract Impact Study provides evidence that cases with cataract are poorer than controls, and that operating on the cataract may help to alleviate poverty.

\section{THE ASSOCIATION BETWEEN PSYCHOSOCIAL STATUS AND MORTALITY IN OLDER ADULTS: EVIDENCE FROM THE ENGLISH LONGITUDINAL STUDY OF AGEING}

CC Craigs, AO House, J Hewison, RM West. Leeds Institute of Health Sciences, University of Leeds, Leeds, UK

\section{doi:10.1136/jech.2009.096727s}

Objectives: To evaluate the effect of psychosocial status on mortality risk among non-institutionalised older adults in England, controlling for selected demographic, health and lifestyle factors.

Participants: Cohort of 11392 participants born on or before 29 February 1952 who responded to Wave 1 of the English Longitudinal Study of Ageing (ELSA), collected in 2002, and who participated in the Health Survey for England (HSE) survey in years 1998, 1999, or 2001

Methods: The following three measures of psychosocial health, which were assessed at Wave 1, were included in the analysis: CASP-19, a measure of quality of life in early old age; the 12 item General Health Questionnaire (GHQ-12); and the Center for Epidemiologic Studies Depression Scale (CES-D). The number of negative statements agreed with or positive statements disagreed with were totalled separately for each of the three measures, resulting in scores in the range 0 to 19 for CASP-19, 0 to 12 for GHQ-12, and 0 to 8 for CES-D. Mortality status up to December 2006, as reported by ELSA in the Index file, was obtained from the Office for National Statistics. Logistic regression modelling was performed separately for each of the three measures, controlling for the following variables assessed at Wave 1: age, sex, marital status, highest educational qualification, smoking status, alcohol consumption, and self reported long-standing illness, disability and infirmity. The analysis was then replicated with a latent construct measured by CASP-19, GHQ-12, and CES-D.

Results: A total of 703 participants had died up to December 2006. The mean scores for participants who were identified as alive 